\title{
DRIVE-BY BRIDGE PARAMETER IDENTIFICATION: AN OVERVIEW
}

\author{
J.T. LI and X.Q. ZHU* \\ School of Civil and Environmental Engineering, University of Technology Sydney, NSW 2007, Australia \\ Emails: Jiantao.li@student.uts.edu.au,xinqun.zhu@uts.edu.au \\ *Corresponding author
}

\begin{abstract}
Drive-by bridge parameter identification using an instrumented vehicle has drawn great attention in the field of bridge structure health monitoring. The feasibility of the drive-by inspection methods has been verified by a number of studies. However, most of the studies are conducted with numerical simulations and the successful field application for bridge parameter identification requires more factors to be taken into consideration. Influences from road surface roughness, vehicle moving speed, uncertainties in vehicle models and measurement noise etc., may make the drive-by parameter identification challenging and impede its practical application. Moreover, the time variant features of the vehicle-bridge interaction (VBI) system is another important factor for the drive-by bridge parameter identification which needs further investigation to ensure the accuracy of the identified results. This paper presents a brief overview on the drive-by bridge parameter identification of the authors' recent experimental investigation to show the feasibility and challenges for the practical implementation of drive-by bridge parameter identification.
\end{abstract}

Keywords: drive-by parameter identification; vehicle-bridge interaction; time-frequency analysis.

\section{INTRODUCTION}

Due to the fact that a large amount of bridge infrastructures are subjected to gradual deterioration and structurally deficient, monitoring the condition of these bridges is very important. Bridge parameter identification is a critical component in bridge structure health monitoring. The measured bridge modal parameters, such as the modal frequencies, damping and mode shapes can be effective indicators of the structure degradation and damage. Conventional modal parameter identification requires extensive instrumentation on the bridge structure to get the dynamic responses from sensors on different locations of the bridge (Carden and Fanning, 2004). This direct approach can pose a number of practical problems, such as the need of bridge closure, accidental damage to installed equipment, the high initial and recurring cost of the sensory system, etc. The idea of an indirect approach, also referred as drive-by inspection, was proposed by Yang et al. (2004) that the dynamic parameters of bridge were extracted from the response of a passing instrumented vehicle. Without requirement of bridge instrumentation, the drive-by inspection technique is convenient, efficient and low-cost. Over the past decade, a great deal of research has been conducted in the field of indirect bridge modal identification. Besides the frequency identification, the indirect approach was also extended to the estimation of bridge damping and mode shapes. González et al. (2012) and Keenahan et al. (2014) identified the bridge damping using a moving instrumented vehicle or a trailer-truck. The vehicle response had also been used for the bridge mode shape identification (Yang et al., 
2014; Malekjafarian and OBrien, 2017). A review paper was presented by Zhu and Law (2015) for structural health monitoring based on the VBI data. Bridge identification techniques using vehicle responses and both vehicle and bridge responses were reviewed. Malekjafarian et al. (2015) and Yang and Yang (2018) presented critical review of indirect methods for bridge modal parameter identification and the challenges for successful implementation of drive-by inspection in practice. Recently, some experimental investigations have been conducted by the authors to identify the bridge modal parameters using responses of vehicle models in the lab which is presented in this paper. The VBI system is time-variant with the operational variations of traffic excitation on the structure. Time-frequency characteristic of a VBI system due to the interaction is important factor for the accurate bridge parameter identification. A recently proposed time-frequency (TF) analysis method, synchroextracting transform (Yu et al., 2017), is used to extract the time-varying characteristic of the VBI system. The instantaneous frequency trajectories of a filed cable-stayed bridge under the excitation of a truck are extracted to show the time-varying frequency features.

\section{BRIDGE MODAL PARAMETER IDENTIFICATION}

\subsection{VEHICLE-BRIDGE INTERACTION SYSTEMS}

Considering a VBI system as shown in Figure 1, the vehicle is modelled as a single-degreeof-freedom quarter-car model and the bridge deck is simulated as a simply-supported beam. The coupled equation of motion of the system can be written as

$$
\mathbf{M} \ddot{\mathbf{d}}+\mathbf{C} \dot{\mathbf{d}}+\mathbf{K d}=\mathbf{F}
$$

where $\mathbf{M}, \mathbf{C}$, and $\mathbf{K}$ are the mass, damping and stiffness matrices of the system, respectively; $\mathbf{F}$ is the force vector and $\mathbf{d}$ is the response vector of the system. The vehicle response contains dynamic information of the bridge. Thus, the bridge modal parameters can be estimated from the vehicle response.

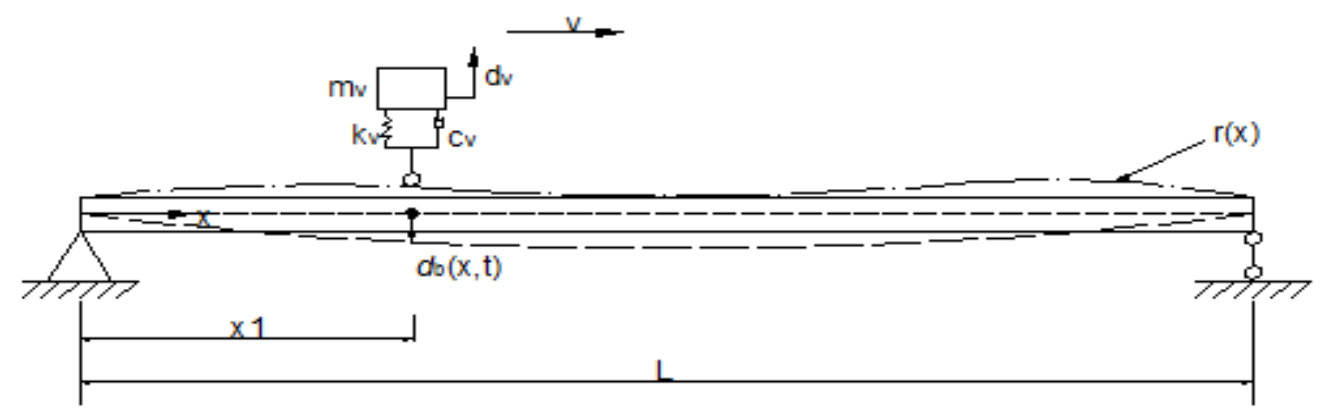

Figure 1 Vehicle and bridge interaction model

\subsubsection{Identification of bridge modal frequencies and mode shapes using SSI}

With the premise that the multiple excitations to the bridge were modelled as white noise excitation to the bridge supports, stochastic subspace identification (SSI) based technique was adopted by $\mathrm{Li}$ et al. (2019b) to identify bridge modal frequencies and mode shapes with instrumented vehicles. The bridge frequencies can be easily extracted from responses of moving vehicle when the bridge surface roughness is smooth. For a rough surface, the subtraction of the responses measured from two successive identical vehicles can greatly reduce the effect of road surface roughness. To identified the bridge mode shapes, two instrumented vehicles were used with one remain stationary on the bridge as reference sensor and the other one moving 
over the bridge as a moving sensor. The responses measured from two vehicles were segmented into multi datasets based on the number of selected beam elements for analysis. Within each dataset, the response was assumed stationary due to the short measure time for each signal segment. A short time SSI method with a rescale procedure was proposed to analyze the segmented responses and get the bridge mode shapes.

\subsubsection{Identification of bridge modal frequencies using blind modal identification with SSA}

A novel drive-by blind modal identification with singular spectrum analysis (SSA) was proposed to extract bridge modal frequencies from vehicle response ( $\mathrm{Li}$ et al., 2019a). Only one sensor was used to equip the vehicle and measure vehicle response during its moving over the bridge. The acquired response was first decomposed into a multichannel datasets using SSA, and a blind modal identification method was implemented to analyze the datasets to extract the different response components contained in the vehicle response. The bridge modal frequencies were identified from the corresponding components. The method was found robust to the measurement noise and can identify bridge modal frequencies without requirement of subtraction technique when there is road surface roughness. Hilbert transform could be used to analyze bridge related components and their instantaneous frequencies.

\subsection{Experimental investigation in the lab}

Experimental tests in the lab were conducted to verify the proposed indirect bridge modal identification techniques. A vehicle bridge interaction testbed were built as shown in Figure 2 . The bridge model consists of three rectangular section steel beams. The one in the middle is the main beam with a length of $6 \mathrm{~m}$ and the width and depth of the steel beams are $100 \mathrm{~mm}$ and $15 \mathrm{~mm}$, respectively. The main beam is in a two-span continuous simply-supported configuration. Half-cylinder steel bar and cylinder steel bar are used to simulate the pinned support and roller support to the beam, respectively. A leading beam and a trailing beam are sitting in front of and at the rear of the main beam to allow for acceleration and deceleration of the vehicle. The length of the side beams is $3 \mathrm{~m}$. Vehicle model with different configurations including one and two-axle vehicles were manufactured for study. One-axle vehicle was manufactured to simulate the SDOF vehicle mode used in the analytical program. The two-axle vehicle model runs on four rubber wheels. The one-axle vehicle was connected with the twoaxle vehicle when they were passing over the bridge. The mass of the one- and two-axle vehicles are 2 and $4 \mathrm{~kg}$, respectively. The model vehicles are pulled along the guide with an electric motor.

The modal testing has been carried out on the vehicle and bridge models with an impulse hammer. The first two natural bridge frequencies were obtained as 5.68 , and $8.48 \mathrm{~Hz}$, respectively. Spectrum analysis on the vehicle responses gave the modal frequency of the single-axle vehicle as $29.37 \mathrm{~Hz}$.

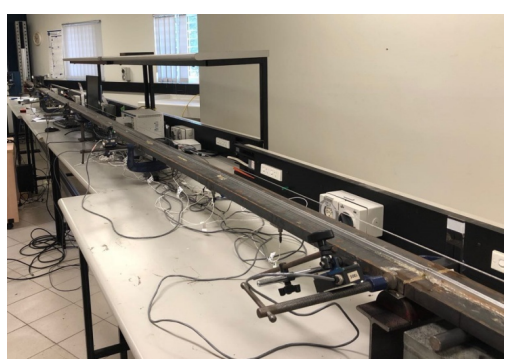

(a) Bridge model

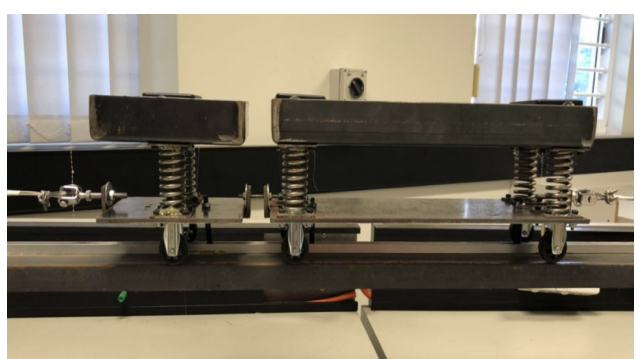

(b) vehicle model

Figure 2 Vehicle bridge interaction test model in the lab 
In drive-by bridge modal parameter identification test, a wireless sensory system was setup and wireless accelerometers were used to measure the responses. For the bridge mode shape identification using SSI based method, a wireless sensor was installed on the single-axle vehicle as a moving sensor with the other one installed on the beam (at $1.1 \mathrm{~m}$ to the right support) as the reference sensor. The vehicles were pulled with three different speeds at approximately 0.10 , 0.14 and $0.21 \mathrm{~m} / \mathrm{s}$, respectively which were denoted respectively by $\mathrm{v} 1$, v2 and $\mathrm{v} 3$. Measurements from the moving and stationary sensors were analyzed with the proposed method to identify the first mode shape of the beam. Each signal was divided into eight segments. The identified operational mode shape values were compared with those from modal testing using MAC values. Figure 3 shows the results for three different moving speeds. The MAC value decreases from about 0.98 to 0.94 when the speed increases from 0.10 to $0.21 \mathrm{~m} / \mathrm{s}$. The accuracy of the identification is acceptable.

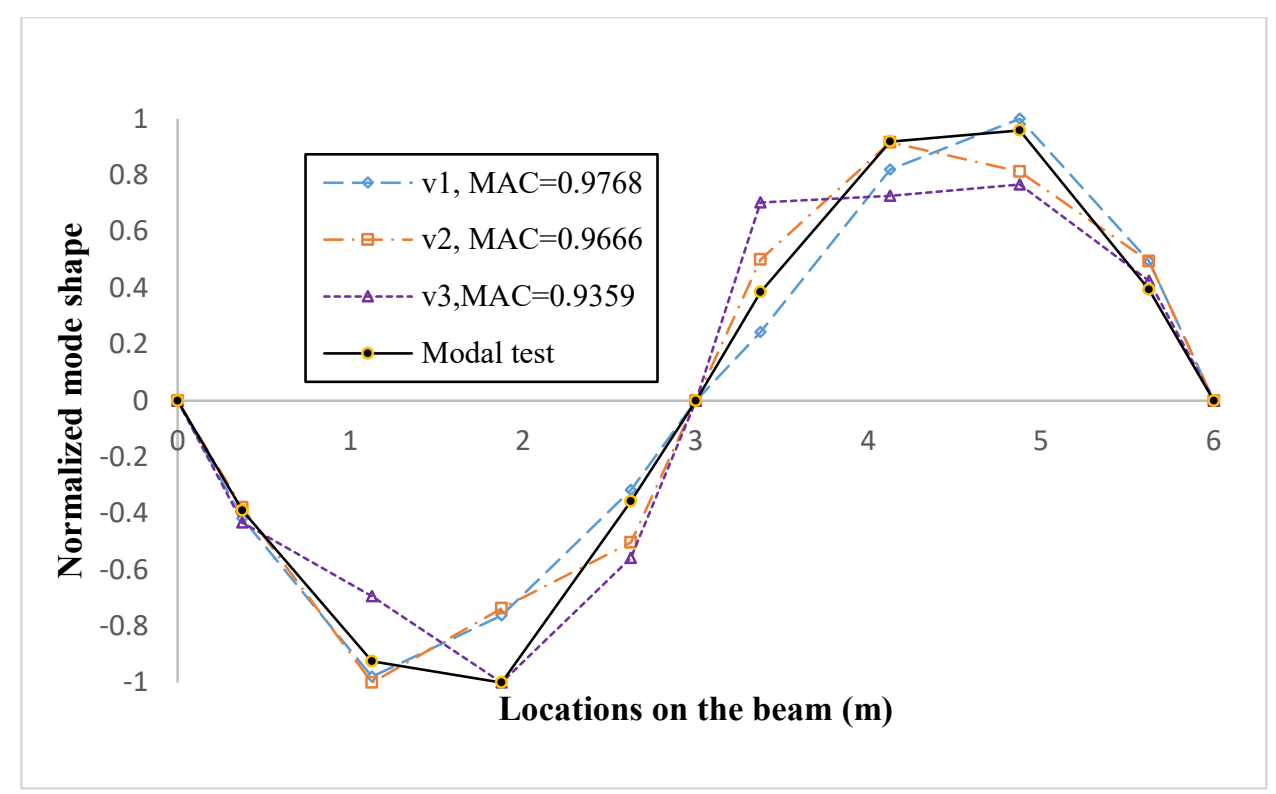

Figure 3 Identified mode shapes with different moving speeds

For the verification of proposed blind modal identification with SSA, only the response from the single-axle vehicle was used. The decomposed components using proposed method are presented in Figure 4 when the speed was $0.10 \mathrm{~m} / \mathrm{s}$. The first component has the frequency $5.21 \mathrm{~Hz}$ that is close to the first modal frequency of the bridge. Similarly, the frequency $8.32 \mathrm{~Hz}$ in Figure 4(b) is related to the second bridge mode. The vehicle modal frequency is identified in the third component as $29.35 \mathrm{~Hz}$. 

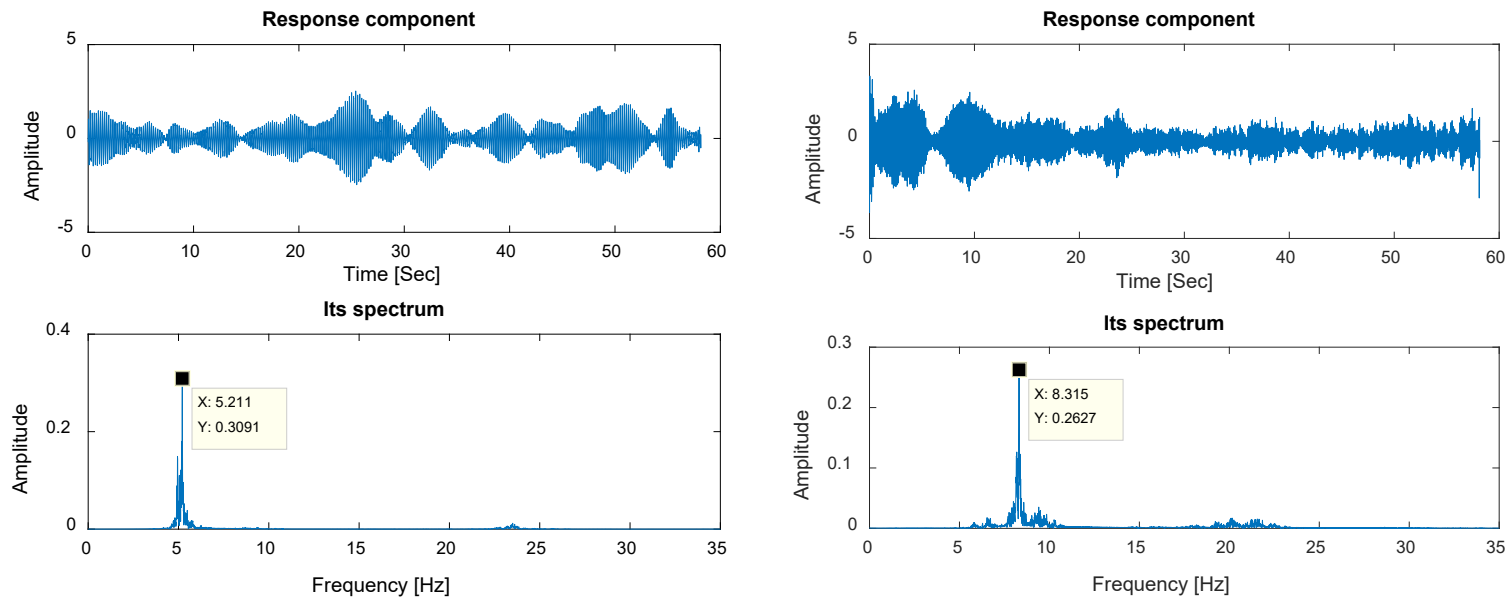

(a) First component and its spectrum

(b) Second component and its spectrum
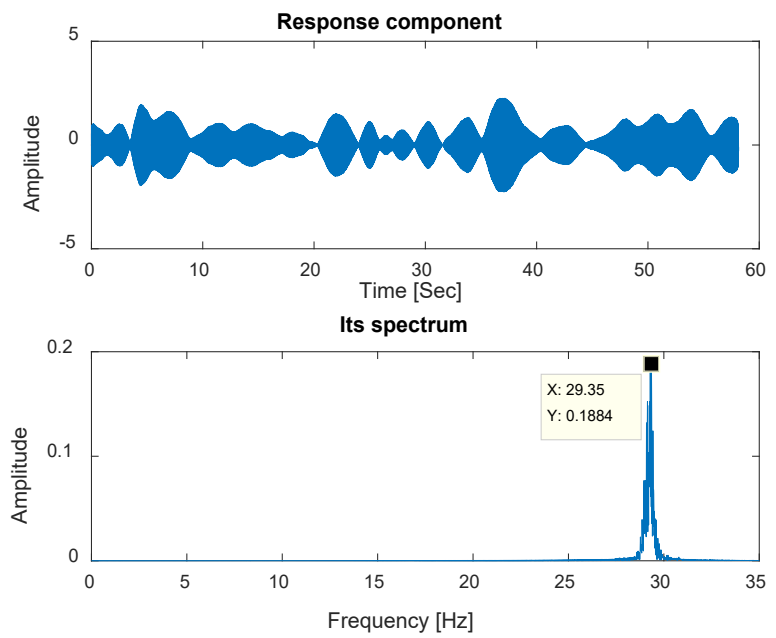

(c) Third component and its spectrum

Figure 4 Decomposed components and their spectra from vehicle response

\section{TIME-FREQUENCY ANALYSIS ON THE BRIDGE RESPONSES UNDER VEHICLE EXCITATION}

This section introduces the time-frequency analysis on the responses measured from an actual bridge. Time-varying characteristic of the frequencies due to the vehicle bridge interaction was investigated. A recent proposed time-frequency (TF) analysis method termed synchroextracting transform (SET) was used to get the instantaneous frequency (IF) trajectories. A short-span cable-stayed bridge over the Great Western Highway (as shown in Figure 5) in New South Wales, Australia was tested. It is a single lane highway bridge with a span $46 \mathrm{~m}$ and a width $6 \mathrm{~m}$. A long-term monitoring system has been installed on the bridge. This system provides an integrated and reliable device to log high-quality data with 24-bit resolution with bandwidth capability of $0-3 \mathrm{kHz}$. The hardware combines instrument excitation, 
voltage regulation, digitization, anti-aliasing filters, and data logging. The logging software is Catman Version 5, which collects all channels at a default sample rate of $600 \mathrm{~Hz}$ (Sun et al., 2017). A dense array of strain gauges and the accelerometers were installed under the bridge deck and timely synchronized to measure the structure responses. Figure 6 shows the accelerometer array on the deck. Modal analysis using the acceleration measurements from sensors on the deck was conducted under ambient excitation. A bridge vibration mode with the frequency $2.00 \mathrm{~Hz}$ was identified as shown in Figure 7 . It can be seen the vibration is related to the vertical bending mode.

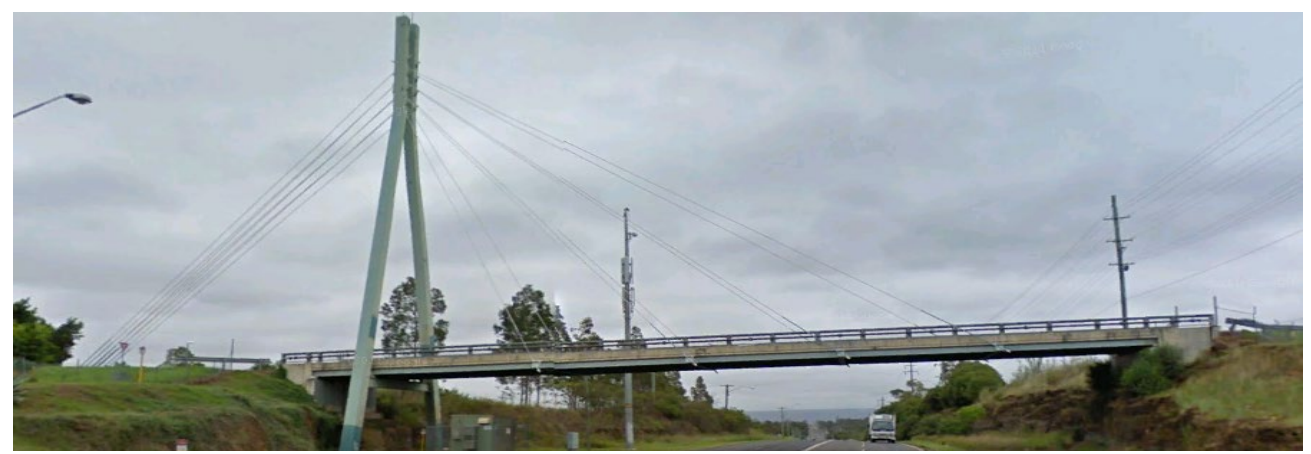

Figure 5 Cable-stayed bridge for tests

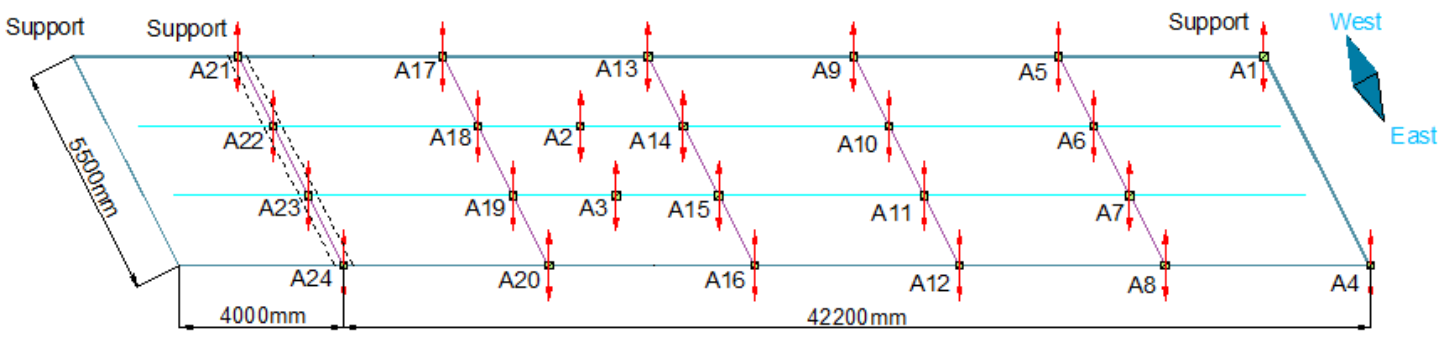

Figure 6 Accelerometer array on the deck

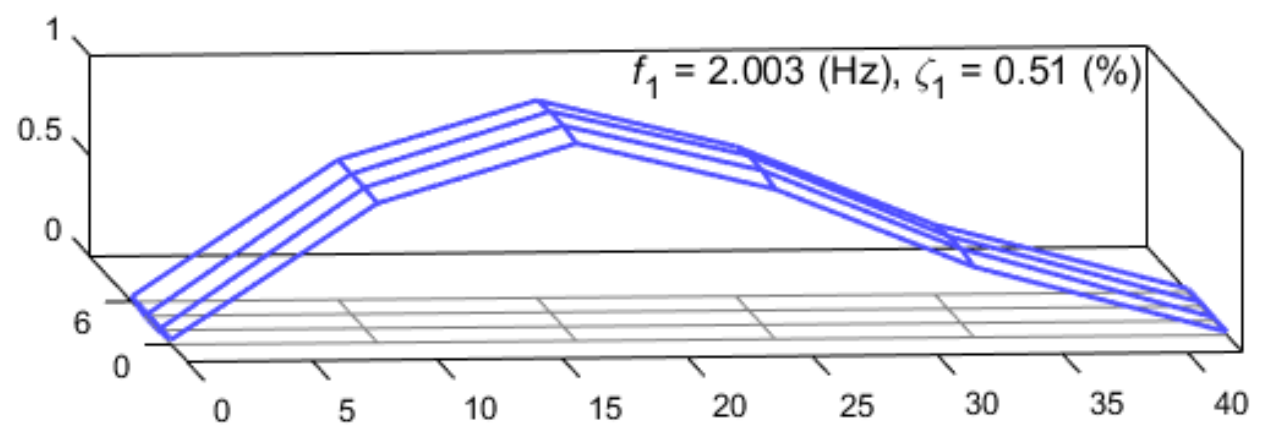

Figure 7 The measured bridge modal parameters under ambient excitation

Responses under different traffic conditions at sensor location A10 were measured and used for analysis. The acceleration responses and the spectra are presented in Figure 8. When no traffic on bridge, only the first bridge frequency can be identified in the response spectrum. For the condition with a moving vehicle on top of the bridge, the vehicular excitation makes the higher bridge vibration modes more prominent. The TFA results of the responses under different traffic conditions are presented in Figure 9. For the condition of without traffic on bridge, the IF 
trajectory of the first bridge vibration mode show little variation. For the conditions of with moving vehicle, the IF trajectories corresponding to the bridge vibration modes show great variations. For the first three modes, the frequency variation becomes very small, while the frequency of the $4^{\text {th }}$ bridge vibration mode varies periodically after about 6 seconds. The composite effects of vehicle and cables account for the complicated variation behaviors of the bridge frequencies.

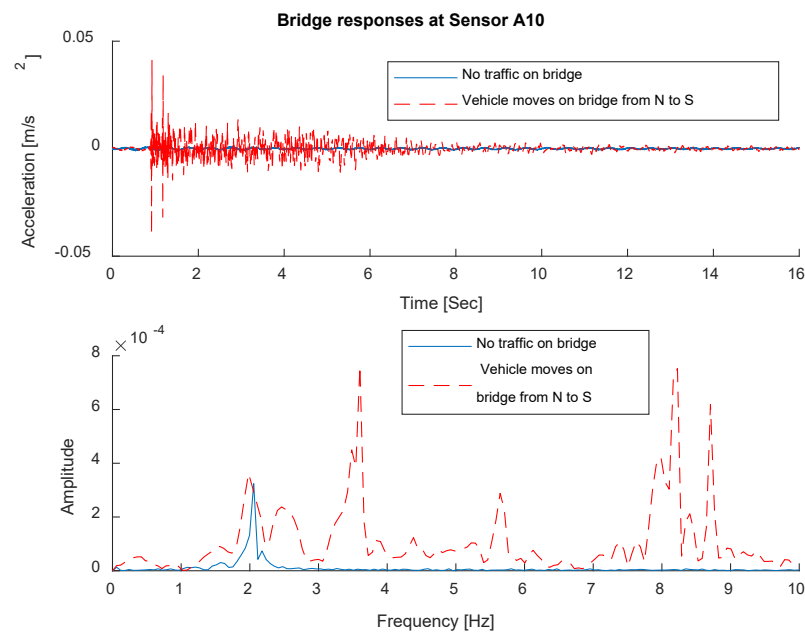

Figure 8 Bridge response measured at sensor A10 and response spectrum

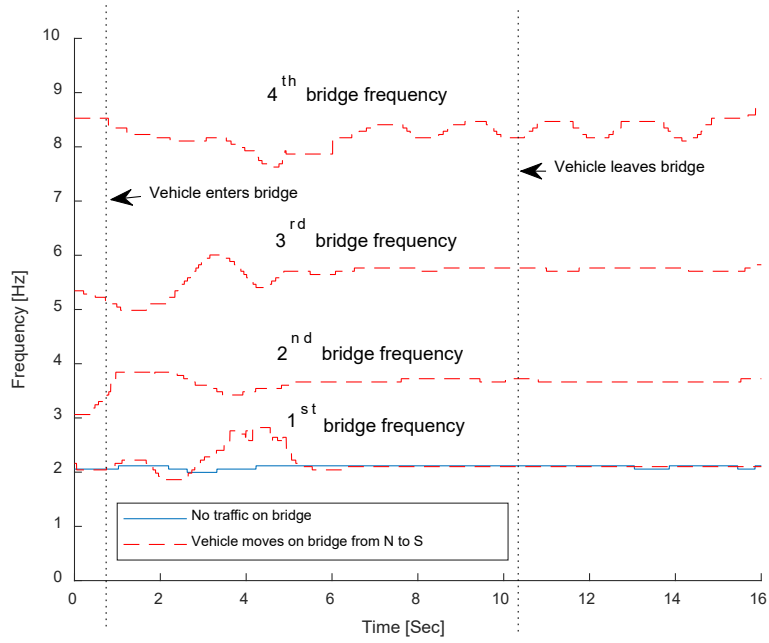

Figure 9 IF trajectories of responses for different traffic conditions

\section{CONCLUSIONS}

This work presents a concise overview on the authors' recent study on the drive-by bridge parameter identification. Using instrumented vehicles, the bridge frequencies and mode shapes can be identified with stochastic subspace identification based method under the assumption of white-noise excitation. With SSA based blind modal identification method, the response components can be extracted from a set of vehicle responses. The bridge frequencies can be estimated from the bridge related components, further study will focus on the identification of other modal parameters from the extracted components. A time-frequency analysis on the bridge responses under heavy traffic excitation reveals the time-varying characteristics of the 
vehicle-bridge interaction system. Further investigation on the bridge damage detection using the time-varying features is recommended.

\section{ACKNOWLEDGEMENTS}

This research is supported by research funding of the Australian Research Council Discover Project (DP160103197). The financial aid is gratefully acknowledged. The data from the actual bridge is measured by Data61 which is greatly acknowledged.

\section{REFERENCES}

Carden EP and Fanning P. (2004) Vibration based condition monitoring: a review. Structural Health Monitoring 3: 355-377.

González A, OBrien EJ and McGetrick P. (2012) Identification of damping in a bridge using a moving instrumented vehicle. Journal of Sound and Vibration 331: 4115-4131.

Keenahan J, OBrien EJ, McGetrick PJ, et al. (2014) The use of a dynamic truck-trailer driveby system to monitor bridge damping. Structural Health Monitoring 13: 143-157.

Li J, Zhu X, Law S-s, et al. (2019a) Drive-By Blind Modal Identification with Singular Spectrum Analysis. Journal of Aerospace Engineering ASCE 32: 04019050.

Li JT, Zhu XQ, Law SS, et al. (2019b) Indirect bridge modal parameters identification with one stationary and one moving sensors and stochastic subspace identification. Journal of Sound and Vibration 446: 1-21.

Malekjafarian A, McGetrick PJ and OBrien EJ. (2015) A review of indirect bridge monitoring using passing vehicles. Shock and Vibration 286139.

Malekjafarian A and OBrien EJ. (2017) On the use of a passing vehicle for the estimation of bridge mode shapes. Journal of Sound and Vibration 397, 77-91.

Sun M, Makki Alamdari M and Kalhori H. (2017) Automated operational modal analysis of a cable-stayed bridge. Journal of Bridge Engineering ASCE 22: 05017012.

Yang YB, Li YC and Chang KC. (2014) Constructing the mode shapes of a bridge from a passing vehicle: a theoretical study. Smart Structures and Systems 13: 797-819.

Yang YB, Lin CW and Yau JD. (2004) Extracting bridge frequencies from the dynamic response of a passing vehicle. Journal of Sound and Vibration 272: 471-493.

Yang YB and Yang JP. (2018) State-of-the-Art Review on Modal Identification and Damage Detection of Bridges by Moving Test Vehicles. International Journal of Structural Stability and Dynamics 18: 1850025.

Yu G, Yu M and Xu C. (2017) Synchroextracting transform. IEEE Transactions on Industrial Electronics 64: 8042-8054.

Zhu XQ and Law SS. (2015) Structural health monitoring based on vehicle-bridge interaction: accomplishments and challenges. Advances in Structural Engineering 18: 1999-2015. 